Moral-García, J.E.; Al Nayf Mantas, M.R.; López-García, S.; Maneiro, R. y Amatria, M. (2019) Nutritional Status and Physical Condition in Active vs. Sedentary Elderly People. Revista Internacional de Medicina y Ciencias de la Actividad Física y el Deporte vol. 19 (76) pp. 685-698 Http://cdeporte.rediris.es/revista/revista76/artestado1102.htm

DOI: $10.15366 /$ rimcafd2019.76.003

\title{
ORIGINAL
}

\section{ESTADO NUTRICIONAL Y CONDICIÓN FÍSICA EN PERSONAS MAYORES ACTIVAS VS. SEDENTARIAS}

\section{NUTRITIONAL STATUS AND PHYSICAL CONDITION IN ACTIVE VS. SEDENTARY ELDERLY PEOPLE}

Moral-García, J.E. ${ }^{1}$; Al Nayf Mantas, M.R. ${ }^{2}$; López-García, S. ${ }^{1}$; Maneiro, R. ${ }^{1}$ y Amatria, M. ${ }^{1}$

${ }^{1}$ Doctor en Ciencias de la Actividad Física y del Deporte. Profesor del departamento de Ciencias Actividad Física y Deporte, Universidad Pontificia de Salamanca (España) jemoralga@upsa.es, slopezga@upsa.es,rmaneirodi@upsa.es,mamatriaji@upsa.es

${ }^{2}$ Graduado en Ciencias de la Actividad Física y del Deporte. Máster en Investigación y Docencia en Ciencias de la Actividad Física y la Salud (España) ralnayfma@gmail.com

Código Unesco / UNESCO Codes: 3206 Ciencias de la Nutrición / Nutrition Sciences; 2411.99 Actividad Física y Salud / Physical activity and health; 3210 Medicina Preventiva / Preventive Medicine; 3212 Salud Pública / Public Health; 6310.09 Calidad de vida / Quality of Life.

Clasificación del Consejo de Europa / Council of Europe classification: 17. Otras (Actividad física y salud; Nutrición) 7 Other (Physical activity; nutrition)

Recibido 15 de febrero de 2015 Received February 15, 2015

Aceptado 5 de mayo de 2018 Accepted May 5, 2018

\section{RESUMEN}

El objetivo del estudio fue conocer la relación entre el estado nutricional, la adherencia a la dieta mediterránea y el nivel de condición física de personas mayores. Participaron 168 personas mayores de 65 años (grupo control=84 personas activas y grupo experimental=84 personas sedentarias). La adherencia a la dieta mediterránea fue medida con el cuestionario MEDIS-FFQ, el nivel de práctica de actividad física mediante una pregunta creada a tal efecto, y las diferentes pruebas físicas con los instrumentos específicos. Los resultados revelaron que el $63.1 \%$ manifestó baja adherencia a la dieta mediterránea y el $34.5 \%$ alta, teniendo los sujetos sedentarios mayor adherencia que los activos ( $p \leq 0.05 ; 46.4 \%$ vs. $22.6 \%$ ). Los sujetos activos tienen mejor condición física que los sedentarios $(p \leq 0.001)$. Por tanto, la mayor parte de las personas mayores 
deben incrementar su adherencia a la dieta mediterránea y la práctica de actividad física como mecanismo de mejora de su salud.

PALABRAS ClAVE: Dieta mediterránea, Actividad física, Salud, Personas mayores.

\begin{abstract}
The objective of the study was to know the relationship between nutritional status, adherence to the Mediterranean diet and the level of physical displacement of older people. 168 people older than 65 years participated (control group $=84$ active people and experimental group $=84$ sedentary people). Adherence to the Mediterranean diet was measured with the MEDISFFQ questionnaire, the level of physical activity practice through a question created for that purpose, and the different physical tests with the specific instruments. The results revealed that $63.1 \%$ showed low adherence to the Mediterranean diet and $34.5 \%$ high, according to the sedentary subject's greater adherence than the active ones ( $p \leq 0.05,46.4 \%$ versus $22.6 \%)$. Active subjects have better physical income than sedentary people $(p \leq 0.001)$. Therefore, most elderly people want to increase their adherence to the Mediterranean diet and the practice of physical activity as a mechanism to reduce their health.
\end{abstract}

KEYWORD: Mediterranean diet, Physical activity, Health, Older people.

\title{
1. INTRODUCCIÓN
}

En la actualidad existe un proceso progresivo de envejecimiento poblacional lo cual supone un incremento constante de personas con dependencia en décadas posteriores (García, Graciani, Guallar, López, Rodríguez y Rodríguez, 2015). La calidad de vida que es definida como el estado de bienestar subjetivo asociado a una buena salud y felicidad (Huiguita, Arias y Antonio, 2015), considerando todos los ámbitos de la persona (Espada y Calero, 2015), siendo un objetivo a alcanzar por este grupo poblacional, por ello la realización de ejercicio físico en personas mayores ha experimentado un auge en las últimas décadas, mediante los programas de actividad física (AF) específicos para mayores que ayudan a que se mantengan sanos (Castañer, Süch, Prat, Camerino y Anguera, 2016), mejorando su movilidad y minimizando el riesgo potencial de sufrir caídas o accidentes (Sherrintong, Fairball, Kirkham, Clemson, Howard, Vogler y Sonnabend, 2016), lo cual puede contribuir a la mejora de la calidad de vida de los mayores (Lera-López, Garrues, Ollo-López, Sánchez, Cabasés y Sánchez, 2017).

Por otro lado, la condición física, que determina la capacidad de afrontar una AF de la manera más eficiente (Duran, Orbegoz, Uribe-Rodríguez y Uribe, 2008), es el factor a desarrollar y para ello existen diferentes propuestas de programas de 
AF para personas mayores, siendo necesario que sean específicos para este colectivo, donde se trabajen cualidades como la resistencia, el equilibrio, aplicando un entrenamiento funcional, con una frecuencia de 2-3 veces por semana y con una duración de aproximadamente 60 minutos por sesión (Weening-Dijksterhuis, Greef, Scherder, Slaets y Van Der Schans, 2011). Otras propuestas incluyen el entrenamiento de fuerza con cargas, por las mejoras detectadas, siendo más eficaz que el entrenamiento aeróbico a la hora de frenar el declive cognitivo y motor (Bossers, Vand Der Woude, Boersma, Horobágyi, Scherder y Van Heuvelen, 2015) o mejorar la densidad mineral ósea de todo el cuerpo (Hinton, Nigh y Thyfault, 2015).

La influencia de la condición física sobre la calidad de vida se puede ver ayudada cuando se conjugan la práctica habitual de AF y una alimentación adecuada, la cual puede contribuir a retrasar el envejecimiento (Scarmeas, Gu, Schupf, Lee, Luchsinger, Stern y Honing, 2014), incrementando la esperanza de vida (De La Montaña, Areal y Míguez, 2009), y ayuda a controlar el exceso de peso, reduciendo potenciales problemas de movilidad asociados a un índice de masa corporal (IMC) tendente a la obesidad (Hergenroeder, Brach, Otto, Sparto y Jakicic, 2011). Orientados en este sentido otros estudios hablan de la dieta mediterránea (DM) como alternativa saludable de alimentación que previene las enfermedades (Martínez, 2016), primando el consumo de alimentos ricos en fibra (Martínez, Veiga, cobo y Carbajal, 2011) y productos vegetales (Escaffi, Miranda, Alonso y Cuevas, 2016), como verduras, legumbres, frutas, frutos secos y cereales como fuentes de antioxidantes, a lo que sumar la ingesta moderada de vino (Trichopoulou, Costacou, Vamia y Trichopoulos, 2003). Mientras que a su vez se reduce el consumo de carnes o azúcares (Standard, 2016). Esta propuesta de alimentación asegura un óptimo aporte calórico y nutricional, previniendo problemas como el cáncer, lo que habitualmente se traduce en un aumento de la longevidad y mayor calidad de vida (Lagiou, Trichopoulos, Sandon, Lagiou, Mucci, Wollk et al., 2006). Aunque en ocasiones las enfermedades o el bajo nivel socioeconómico pueden modificar negativamente la adherencia a la misma (Viñuales, Viñuales, Puzo y Sanclemente, 2015).

El objetivo de este estudio es doble: 1) Conocer el estado nutricional y nivel de condición física de personas mayores españolas y su relación con hábitos saludables; 2) analizar si la participación en programas de AF se relaciona con mayor adherencia a la dieta mediterránea y mejores valores en velocidad de la marcha, fuerza y equilibrio.

\section{MATERIAL Y MÉTODO}

\subsection{Muestra}

Este es un estudio longitudinal de carácter observacional y no intervencionista del estado nutricional y condición física en personas mayores. La muestra total fue de 168 personas mayores de 65 años, de las cuales 84 personas son catalogadas como activas (GE: grupo experimental) puesto que están inscritas 
a programas de AF y deporte que se llevan a cabo en centros de día de ámbito urbano, durante 6 meses de duración, en base al modelo de sesión establecido por Matos-Duarte, Martínez de Haro, Sanz-Arribas, Andrade y Chagas (2017). Las otras 84 personas son completamente sedentarias ya que no realizan ninguna actividad físico-deportiva.

El periodo objeto de análisis comprende desde septiembre de 2016 hasta abril de 2017, ambos inclusive, donde se llevó a cabo el periodo de inclusión de los participantes en el programa de AF. Posteriormente, en mayo de 2017 se hizo la toma de datos de las diferentes pruebas. Para la selección de la muestra se aplicó un muestreo aleatorio simple, evitando posibles azarosos sobre los resultados. Se realizó un filtrado previo del estado físico (descartando los sujetos con un moderado-elevado nivel de dependencia) y a nivel educativo-cultural (siendo descartados las personas que no sabían leer o escribir correctamente) que asegurase un nivel de autonomía suficiente para el desarrollo de las distintas pruebas. Se trabajó con un nivel de confianza del 95\% y un margen de error del $3 \%$. Las características sociodemográficas de la muestra determinan que el $76.8 \%$ son mujeres, todos los participantes tenían más de 65 años (73.57士5.85), el estado civil determina que la mayor parte está casado (53\%), seguidos de viudos $(26.8 \%)$, solteros $(18.5 \%)$ y separados $(1.8 \%)$. Y en referencia al nivel de práctica de AF el $50 \%$ hace, al menos 150 minutos semanales de AF y el restante permanece sedentario sin hacer ninguna actividad físico-deportiva.

\subsection{Instrumento}

Se utilizaron dos cuestionarios para analizar la adherencia a la DM, la frecuencia de consumo de alimentos y el nivel de práctica de AF, empleándose diferentes instrumentos para analizar parámetros relacionados con la condición física. También se recogió información de aspectos sociodemográficos como género, edad, localidad de residencia y estado civil.

a) Para valorar el grado de adherencia a la DM se empleó el cuestionario de Prevención con dieta Mediterránea (PREDIMED), de 14 ítems y previamente validado en población española (Papadaki y Scott, 2002). El cual los participantes debían cumplimentar en base a sus hábitos alimenticios del último año. Para posteriormente comparar el consumo de los distintos alimentos con las directrices marcadas por la Fundación Dieta Mediterránea.

b) Para estudiar la frecuencia de consumos de alimentos se empleó el cuestionario de alimentación validado para población mayor residente en países mediterráneos MEDIS-FFQ (Zaragoza, Ferrer, Cabañero, Hurtado y Laguna, 2014). El cual los participantes debían cumplimentar en base a sus hábitos alimenticios del último año.

c) Para evaluar el nivel de práctica de AF, se utilizó un cuestionario en el cual los participantes eran preguntados sobre la frecuencia semana de 
realización de AF. En el presente estudio se divide a los participantes en 2 grupos: el primer grupo llamado "sedentario" en el que englobamos a aquellos sujetos cuya práctica física es inferior a 150 minutos semanales. En el segundo grupo denominado "activo" se introducen los sujetos que realizan AF por encima de los 150 minutos semanales. Esta división se realiza en base a las recomendaciones estipuladas por la OMS (Organización Mundial de la Salud, 2010) sobre AF, que indica 150 minutos semanales como la cantidad mínima recomendada.

d) Se midió la altura de los participantes con el tallímetro portátil seca 214.

e) Se analizó la composición corporal mediante dos métodos: el IMC (peso/talla (metros) ${ }^{2}$, ajustados a edad y sexo correspondiente. El otro instrumento utilizado fue la tanita, en concreto el medidor de composición corporal modelo BC-418-MA (instrumento que también aporta el peso de los sujetos, empleado para el IMC).

f) Para evaluar la velocidad de la marcha (VM) se utilizaron como instrumentos de medida 2 células fotoeléctricas Microgate kit racetime2 light radio (Microgate, Bolzano, Italia). Los participantes se situaban de pie dos metros antes de la línea de salida, a la señal del investigador tenía que comenzar a andar a la mayor velocidad posible pero sin llegar a correr e ir atravesando las células fotoeléctricas, que medían el tiempo transcurrido entre el paso de unas a otras. La longitud del tramo evaluado fue de 4 metros, distancia extraída de la batería BCF específica para mayores (Avila, Gray y Payete, 2006), y cada sujeto tenía que recorrerlo 3 veces descansando entre cada intento 1 minuto. Al finalizar los evaluadores registraban el mejor tiempo de los 3 medidos.

g) Las mediciones de equilibrio se obtuvieron a partir de un test de equilibrio monopodal empleado (Da Silva, Bilodeau, Parreira, Teixeira y Amirim, 2013) en el cual los participantes debían aguantar el máximo tiempo posible estable sobre una sola pierna mientras la otra permanecía elevada posteriormente formando la articulación de la rodilla un ángulo de $90^{\circ}$. Para ello contaron con 3 intentos con descanso de 5 minutos entre cada uno de ellos. Posteriormente el investigador seleccionaba la mejor de las marcas.

h) La fuerza del tren inferior se estimó mediante el test de la silla empleado en la batería BCF (Avila, Gray y Payete, 2006). En esta prueba los sujetos partían en bipedestación y debían levantarse y sentarse completamente en una silla 5 veces en el menor tiempo posible. Para ello disponían de 3 intentos (seleccionando el mejor de ellos) separados 3 minutos entre sí para poder descansar.

i) Para la medida de la fuerza de prensión manual, se ha utilizado un dinamómetro manual adaptable Grip Strength Dynamometer TKK.5101.Grip-D. Con precisión de $0.1 \mathrm{Kg}$., donde tenían que conseguir 
ejercer la mayor fuerza posible en el instrumento. El sujeto en bipedestación y con los brazos extendidos y separados lateralmente del tronco realizaba cada uno de los 3 intentos de los que disponía seleccionando el mejor de ellos para la investigación.

\subsection{Procedimiento}

La totalidad de las pruebas fue evaluada por el mismo investigador. Cada participante tuvo que realizar una sesión de familiarización con las pruebas con el objetivo de minimizar errores por culpa del desconocimiento sobre las mismas. La participación fue totalmente voluntaria y anónima. Los sujetos no recibieron ninguna compensación económica por su contribución y todos ellos firmaron un consentimiento informado aprobando su participación. La investigación fue desarrollada siguiendo las directrices éticas de la Declaración de Helsinki vigente, cumpliendo en todo momento con los máximos estándares de seguridad y ética profesional para este tipo de trabajos.

\subsection{Análisis de datos}

Se realizó análisis descriptivo de datos mediante análisis de frecuencias, utilizándose la prueba Chi-cuadrado para analizar las diferencias de proporciones entre la variable categórica grupo (control-experimental) y la adherencia a la DM. También se realizó análisis de varianza ANOVA para analizar las diferencias entre la variable categórica grupo y las variables continuas (velocidad de la marcha, fuerza y equilibrio monopodal). Todos los datos fueron tratados de forma anónima mediante un sistema de códigos, empleándose para todos los resultados un nivel de confianza del 95\% $(p<.05)$. Los análisis se realizaron con el programa estadístico SPSS, v. 21.0 para WINDOWS (SPSS Inc., Chicago, EE. UU.).

\section{RESULTADOS}

La tabla 1 refleja los datos relativos al complimiento de las recomendaciones alimentarias en base al patrón establecido en la pirámide de la DM de Fundación Dieta Mediterránea, seguido en un reciente estudio (Zaragoza, Ferrer, Cabañero, Hurtado y Laguna, 2014), relacionados con el GC y GE en personas mayores de Salamanca. En general, existe un alto seguimiento de las recomendaciones en el consumo de alimentos, sobre todo en legumbres (96.4\%), patatas (90.5\%), aceite $(89.9 \%)$, pescado $(83.9 \%)$ y cereales $(72.6 \%)$. Por el contrario, bajos niveles de seguimiento se encuentran en carne procesada $(8.3 \%)$ y carne roja (14.9). Según el grupo de pertenencia existen diferencias significativas $(p \leq 0.05)$, presentando mayor cumplimiento el GE que en el GC, en alimentos como frutas (72.6 vs. 32.1), aceite (97.6 vs. 82.1) o frutos secos (48.8 vs. 32.1). En cambio, obtiene el GC mayor seguimiento que el GE en consumo de verduras (89.3 vs. 67.9 ) o huevos (46.4 vs. 8.3). 
Tabla 1. Cumplimiento con las recomendaciones según la pirámide de DM de la Fundación de Dieta Mediterránea y grado de adherencia a la DM según el cuestionario PREDIMED. Clasificación por nivel de práctica de AF (GC y GE).

\begin{tabular}{|c|c|c|c|c|}
\hline & \multicolumn{2}{|c|}{ Grupo de pertenencia } & \multirow[b]{2}{*}{$p$} & \multirow{2}{*}{$\begin{array}{c}\text { Muestra } \\
\text { Total } \\
n(\%)\end{array}$} \\
\hline & $\begin{array}{c}\mathrm{GC} \\
n(\%)\end{array}$ & $\begin{array}{l}\text { GE } \\
n(\%)\end{array}$ & & \\
\hline \multicolumn{5}{|l|}{$\begin{array}{l}\text { Frecuencia consumo alimentos } \\
\text { (extraídos del cuestionario MEDIS-FFQ) }\end{array}$} \\
\hline \multicolumn{5}{|l|}{ Consumidos dos veces al día } \\
\hline Frutas & $27(32.1)$ & $61(72.6)$ & .000 & $88(52.4)$ \\
\hline Verduras & $75(89.3)$ & 57 (67.9) & .001 & $132(78.6)$ \\
\hline Aceite & $69(82.1)$ & $82(97.6)$ & .001 & $151(89.9)$ \\
\hline Cereales & $60(71.4)$ & $62(73.8)$ & .863 & $122(72.6)$ \\
\hline \multicolumn{5}{|l|}{ Consumidos cada día } \\
\hline Lácteos y derivados & $48(57.1)$ & $46(54.5)$ & .877 & $94(56.0)$ \\
\hline Frutos secos & $27(32.1)$ & $41(48.8)$ & .041 & $68(40.5)$ \\
\hline \multicolumn{5}{|l|}{ Consumidos semanalmente } \\
\hline Carne blanca (2 raciones) & $42(50)$ & $28(33.3)$ & 0.42 & $70(41.7)$ \\
\hline Pescado ( $\geq 2$ raciones) & $75(89.3)$ & $66(78.6)$ & .092 & $141(83.9)$ \\
\hline Carne roja (<2 raciones) & $3(3.6)$ & $22(26.2)$ & .000 & $25(14.9)$ \\
\hline Carne procesada ( $\leq 1$ ración) & $9(10.7)$ & $5(6.0)$ & .403 & $14(8.3)$ \\
\hline Huevos (2-4 raciones) & $39(46.4)$ & $7(8.3)$ & .000 & $46(27.4)$ \\
\hline Legumbres & $81(96.4)$ & $81(96.4)$ & 1.00 & $162(96.4)$ \\
\hline Patatas ( $\leq 3$ raciones) & $72(85.7)$ & $80(95.2)$ & .063 & $152(90.5)$ \\
\hline Dulces & $18(21.4)$ & $11(13.1)$ & .220 & 29 (17.3) \\
\hline $\begin{array}{l}\text { Grado de adherencia a la dieta mediterránea } \\
\text { (extraído del PREDIMED) }\end{array}$ & & & .138 & \\
\hline Bajo (0-8) & $45(53.6)$ & $65(77.4)$ & & $110(65.5)$ \\
\hline Alto $(\geq 9)$ & $39(46.4)$ & $19(22.6)$ & & $58(34.5)$ \\
\hline
\end{tabular}

La valoración global de la DM arroja una puntuación media de 6.79 1.98 , de los cuales el $63.1 \%$ manifestó tener una adherencia baja y el $34.5 \%$ alta. Los resultados promedio determinan que las personas mayores del GC $(7.32 \pm 2.16)$

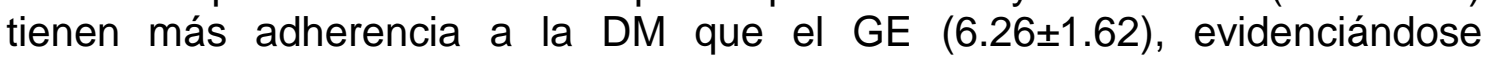
diferencias significativas $(F(2,167)=6.37 ; p \leq 0.01)$.

También ha sido analizado el estado nutricional de las personas mayores, de las cuales el $51.8 \%$ tiene sobrepeso y el $27.4 \%$ obesidad, encontrándose diferencias significativas en los valores promedio en el género $(p=0.043)$, teniendo las mujeres un IMC más elevado que los hombres (28.45 vs. 26.96). Con respecto al tipo de grupo de pertenencia, se han encontrado diferencias significativas $(\mathrm{p}=.001)$ puesto que las personas mayores del GE presentan valores de normopeso más elevados que el GC (32.1\% vs. 9.5\%), incrementándose el sobrepeso y obesidad sobre todo en el GC. En relación al porcentaje de grasa corporal se han encontrado diferencias significativas $(p>0.05)$ por género y tipo de grupo, teniendo los hombres y las personas mayores del GE menores porcentajes de grasa corporal total y en cada uno de los segmentos corporales analizados (tabla 2). 
Tabla 2. Valoración del estado nutricional.

\begin{tabular}{|c|c|c|c|c|c|c|c|}
\hline & & \multicolumn{3}{|c|}{ Comparación por género } & \multicolumn{3}{|c|}{ Comparación por grupo práctica } \\
\hline & & Hombre & Mujer & $\mathrm{p}$ & $\mathrm{GC}$ & GE & $\mathrm{p}$ \\
\hline \multirow{4}{*}{$\geqq$} & Total (promedio) & $26.96 \pm 2.76$ & $28.45 \pm 4.27$ & .043 & $28.56 \pm 3.60$ & $27.64 \pm 4.37$ & .139 \\
\hline & Normopeso (\%) & 23.1 & 20.2 & & 9.5 & 32.1 & \\
\hline & Sobrepeso (\%) & 61.5 & 48.8 & .115 & 60.7 & 42.9 & .001 \\
\hline & Obesidad (\%) & 15.4 & 31.0 & & 29.9 & 25.0 & \\
\hline \multirow{4}{*}{ 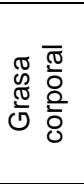 } & MG Total (\%) & $27.88 \pm 5.65$ & $37.43 \pm 6.33$ & .000 & $37.24 \pm 5.84$ & $33.19 \pm 8.19$ & .000 \\
\hline & MG Tronco (\%) & $24.67 \pm 4.72$ & $38.37 \pm 8.31$ & .000 & $36.88 \pm 7.63$ & $33.50 \pm 10.96$ & .021 \\
\hline & MG Pierna D (\%) & $23.56 \pm 5.71$ & $42.83 \pm 5.43$ & .000 & $41.73 \pm 6.90$ & $34.98 \pm 11.12$ & .000 \\
\hline & MG Pierna I (\%) & $30.84 \pm 6.30$ & $33.47 \pm 7.07$ & .039 & $34.19 \pm 6.12$ & $31.54 \pm 7.54$ & .014 \\
\hline
\end{tabular}

MG: masa grasa; D:Derecha; I: Izquierda

Tras el análisis descriptivo de los resultados se puede observar como las personas del GE presentan mejor nivel de condición física en las diferentes pruebas analizadas en comparación al GC. En la tabla 3 se aprecia como son los sujetos del GE tienen una velocidad de la marcha superior al GC (2.463s vs. 10.749s.) $(F(1,166)=147.291 ; p=.000)$, mejores puntuaciones en test de la silla (8.327s. vs. 17.251s.) $(F(1,166)=225.728 ; p=.000)$, más fuerza de prensión manual (15.309 vs. 8.571) $(F(1,166)=46.410 ; p=.000)$, y mejor equilibrio (30.057 vs. 2.516) $(F(1,166)=75.464 ; p=.000)$.

Tabla 3. Análisis descriptivo y de varianza de las diferentes variables analizadas que analizan la condición física según el GC y GE.

\begin{tabular}{|c|c|c|c|c|c|c|c|c|c|}
\hline \multirow{2}{*}{ Prueba } & \multicolumn{4}{|c|}{ Descriptivos } & \multicolumn{5}{|c|}{ ANOVA } \\
\hline & Grupo & Media & $\begin{array}{l}\text { Desv. } \\
\text { típica }\end{array}$ & $\begin{array}{l}\text { Error } \\
\text { típico }\end{array}$ & $\begin{array}{l}\text { Suma de } \\
\text { cuadrados }\end{array}$ & $\begin{array}{c}\text { GI } \\
(D, N)\end{array}$ & $\begin{array}{c}\text { Media } \\
\text { cuadtrática }\end{array}$ & $\mathbf{F}$ & Sig \\
\hline \multirow{2}{*}{$\begin{array}{l}\text { Velocidad } \\
\text { de la } \\
\text { marcha } \\
\text { (seg.) }\end{array}$} & GC & 10.749 & 6.193 & .675 & 2883.661 & \multirow{2}{*}{1,166} & 2883.661 & \multirow{2}{*}{147.291} & \multirow{2}{*}{.000} \\
\hline & GE & 2.463 & .889 & .097 & & & & & \\
\hline \multirow{2}{*}{$\begin{array}{c}\text { Fuerza } \\
\text { tren } \\
\text { inferior } \\
\text { (seg.) }\end{array}$} & GC & 17.251 & 4.829 & .526 & 3344.555 & \multirow{2}{*}{1,166} & 3344.555 & \multirow{2}{*}{225.728} & \multirow{2}{*}{.000} \\
\hline & GE & 8.327 & 2.511 & .273 & 2459.577 & & & & \\
\hline \multirow{2}{*}{$\begin{array}{c}\text { Fuerza } \\
\text { prensión } \\
\text { manual } \\
\text { (kgs.) }\end{array}$} & GC & 8.571 & 6.336 & .691 & 1906.881 & \multirow{2}{*}{1,166} & 1906.881 & \multirow{2}{*}{46.410} & \multirow{2}{*}{.000} \\
\hline & GE & 15.309 & 6.482 & .707 & 6820.524 & & & & \\
\hline \multirow{2}{*}{$\begin{array}{l}\text { Equilibrio } \\
\text { (seg.) }\end{array}$} & GC & 2.516 & 2.576 & .281 & 31857.170 & \multirow{2}{*}{1,166} & 31857.170 & \multirow{2}{*}{75.464} & \multirow{2}{*}{.000} \\
\hline & GE & 30.057 & 28.942 & 3.157 & 70076.881 & & 422.150 & & \\
\hline
\end{tabular}

\section{DISCUSIÓN}

Esta investigación presenta como objetivos fundamentales conocer el estado nutricional y analizar si la participación en programas de AF se relaciona con una mayor adherencia a la dieta mediterránea y mejores valores en velocidad de la marcha, fuerza y equilibrio. En general, más de un tercio de la muestra presenta una alta adherencia a la DM (34.5) un resultado bastante positivo y común en la población de mayor edad, ya que como apuntan (Norte y Ortiz, 2011) la población de edad avanzada habitualmente lleva una dieta más saludable. En 
este estudio el grupo sedentario presento mayor adherencia, al igual que otra investigación donde los sujetos con mayor adherencia a la DM presentaban niveles medios-bajos de AF (Talegawkar et al., 2012). Analizando la ingesta de alimentos, las personas mayores del GE son los que consumen fruta y frutos secos con más frecuencia. Esta tendencia ha sido analizada anteriormente asociándose este consumo a un mayor nivel de práctica de AF (Conklin, Forouhi, Suhrcke, Surtees, Wareham y Monsivais, 2014; Mäkinen et al., 2012). En el caso de los frutos secos (Hernández y Goñi, 2015), un 25\% de los ancianos españoles los consumen semanalmente, datos son similares a los de nuestro GC (32\%) superados significativamente $(\mathrm{p}=.041)$ por de los del $\mathrm{GE}(49 \%)$. Lo cual resulta muy importante por la relación existente entre el consumo de estos alimentos y el descenso de riesgo de sufrir enfermedades cardiacas (Scwingshackl, Hoffmann, Misshach, Stelmach Y Boeing, 2016).

Si examinamos otros ítems de cuestionario, en el GE resalta el consumo inferior a 2 raciones semanales de carne roja y procesada $(26.2 \%$ y $6 \%$ respectivamente), factor que determina una correcta DM (Ortiz, Norte, Zaragoza, Fernández y Davo, 2012). También se ha de tener presente que la promoción del ejercicio puede actuar como incentivo en un aumento de micronutrientes (Martínez, 2016). Tal es así que en el GE se aprecia una alta ingesta de frutas (72.6\%) y verduras (67.9\%), por lo que según algunos autores (Martínez, 2016) son cubiertas las necesidades de micronutrientes tales como vitaminas $A, C$ y $D$ entre otros. Estos datos cobran más relevancia al compararlos con los obtenidos en otro estudio anterior (Martí, Cascales, Martínez, Sánchez y Pérez, 2015), quienes empleando el mismo cuestionario en personas mayores activas observaron una baja ingesta de frutas (15\%) y verduras $(55 \%)$ comparada con la obtenida en el actual estudio. Pero si una ingesta bastante mayor de carnes rojas y procesadas, las cuales ingerían con asiduidad alrededor del $50 \%$ de su muestra.

Uno de los objetivos del presente estudio ha sido el de evaluar la condición física de los participantes. Erigiéndose la velocidad de la marcha como una variable notablemente útil a la hora de predecir el riesgo de defunción (Hardy, Perera, Roumani, Chandler y Studenski, 2007). En el presente estudio se obtuvieron diferencias significativas $(p=.000)$ entre ambos grupos en lo que se refiere a velocidad de la marcha, presentando una mejor velocidad aquellos pertenecientes al GE. Dichos datos concuerdan con otros investigadores quienes además afirman que en personas mayores la velocidad de la marcha incrementa en gran medida con la práctica de AF (Barbat, Varvalho, Rolland, Vellas y Aubertin, 2016). No en vano, se afirma (Dodson et al., 2015) que existe más posibilidad de sufrir accidentes si se tienen niveles de velocidad de la marcha pobres $(-0.8 \mathrm{~m} / \mathrm{s})$.

La fuerza del tren inferior en la actual investigación evidenció diferencias significativas $(p=.000)$ entre ambos grupos, siendo el GE quién presentan mejores valores. Tendencia similar a la encontrada por otros investigadores los cuales hallaron diferencias significativas $(p=.002)$, presentando las personas 
mayores activas mejores puntuaciones (Possamai, Zogo, Boni, Jacques, Dorst y Dorst, 2015).

Por otro lado la fuerza de prensión manual hallada con dinamómetro, manifiesta diferencias significativas ( $p=.000$ ), con resultados favorables al GE con respecto al GC. No obstante, la media del GE (15.3) se sitúa por debajo de la adecuada para Sallinen et al. (2010) quienes hablan de que por debajo de $21 \mathrm{~kg}$. el riesgo de padecer algún problema de movilidad aumenta.

El equilibrio monopodal, refleja diferencias significativas $(p=.000)$, siendo el GE quién tiene mejores valores medios (30.057) en comparación al GC (2.516), obteniendo una puntuación similar a otro studio con personas mayores activas (Da Silva, Bilodeau, Parreira, Teixeira y Amorin, 2013). También se ha comprobado (Nicholson, Mckean y Burkey, 2015) que el equilibrio monopodal de los participantes mayores de 55 años que realizaron un programa basado en trabajo de resistencia mejoró en un $24 \%$ mientras que en el grupo inactivo en el mismo periodo de tiempo se observó un declive de $-7 \%(p=.006)$.

\section{CONCLUSIONES}

Este estudio no estuvo libre de limitaciones, como la posibilidad de que algunos de los ítems o pruebas pudieran ser malinterpretados, con o sin intención, por parte de las personas mayores. Aunque, este riesgo queda reducido, probablemente, debido a que en todo el proceso se garantizó el anonimato de las respuestas emitidas (en el caso del cuestionario), y también porque todas las pruebas fueron ejemplificadas y contaron con la supervisión permanente del investigador responsable. En lo referente a las futuras líneas de investigación afines a la temática, podría resultar interesante incluir una valoración cuantitativa de los hábitos alimentarios, para así compensar las posibles debilidades del cuestionario carente de esta información. De igual manera, podría ser interesante aplicar una batería de test en diferentes momentos, proyectando así un estudio longitudinal de mayor duración.

En conclusión, más de un tercio de las personas mayores presenta niveles óptimos de adherencia a la DM, siendo los sujetos activos (GE) los que secundan más la DM en los niveles medios de adherencia. Además, las personas mayores activas (GE) tienen mejor nivel de condición física que las sedentarias (GC).

\section{REFERENCIAS BIBLIOGRÁFICAS}

Ávila, J.A., Gray, K., \& Payette, H. (2006). Medición de las capacidades físicas de adultos mayores de Quebec: un análisis secundario del estudio NuAge. Salud Pública México, 48(6), 446-454.

Barbat-Artigas, S., Carvalho, L. P., Rolland, Y., Vellas, B., \& Aubertin-Leheudre, M. (2016). Muscle strength and body weight mediate the relationship between physical activity and usual gait speed. Journal of the American 
Medical Directors Association, 17(11), 1031-1036. DOI: https://doi.org/10.1016/j.jamda.2016.06.026

Bossers, W. J., van der Woude, L. H., Boersma, F., Hortobágyi, T., Scherder, E. J., \& van Heuvelen, M. J. (2015). A 9-week aerobic and strength training program improves cognitive and motor function in patients with dementia: a randomized, controlled trial. The American Journal of Geriatric Psychiatry, 23(11), 1106-1116.

Castañer, M., Saüch, G., Prat, Q., Camerino, O., \& Anguera, M.T. (2016). La percepción de beneficios y de mejora del equilibrio motriz en programas de actividad física en la tercera edad. Cuadernos de Psicología del Deporte, 16(1), 77-84.

Conklin, A.I., Forouhi, N.G., Suhrcke. M., Surtees. P., Wareham, N.J., \& Monsivais, P. (2014). Variety more than quantity of fruit and vegetable intake varies by socioeconomic status and financial hardship. Findings from older adults in the EPIC cohort. Appetite, 83, 248-255. DOI: https://doi.org/10.1016/j.appet.2014.08.038

Da Silva, R.A., Bilodeau, M., Parreira, R.B., Teixeira, D.C., \& Amorim, C.F. (2013). Age-related differences in time-limit performance and force platform-based balance measures during one-leg stance. $J$ Electromyography and kinesiology, 23(3), 634-639. DOI: https://doi.org/10.1016/i.jelekin.2013.01.008

De la Montaña Miguélez, J., Salve, C. A., \& Bernárdez, M. M. (2009). Evaluación del riesgo nutricional mediante el MNA en una población anciana no institucionalizada. Archivos Latinoamericanos de Nutrición, 59(4), 390.

Dodson, J. A., Arnold, S. V., Gosch, K. L., Gill, T. M., Spertus, J., Krumholz, H. M., \& Alexander, K. P. (2016). Slow Gait Speed and Risk of Mortality or Hospital Readmission Following Myocardial Infarction in the TRIUMPH Registry. Journal of the American Geriatrics Society, 64(3), 596. DOI: 10.1111/jgs. 14016

Duran, D, Orbegoz Valderrama, L. J, Uribe-Rodríguez, A. F., \& Uribe Molina, J. M. (2008). Integración social y habilidades funcionales en adultos mayores. Univ Psy, 7(1), 263-270.

Escaffi, M. J., Miranda, M., Alonso, R., \& Cuevas, A. (2016). Dieta mediterránea y vitamina $\mathrm{D}$ como potenciales factores preventivos del deterioro cognitivo. Revista Médica Clínica Las Condes, 27(3), 392-400. DOI: https://doi.org/10.1016/j.rmclc.2016.06.012

Espada Mateos, M., \& Calero Cano, J. C. (2015). Salud percibida por las personas mayores en España en función del tipo de demanda de actividad física. Movimento, 21(4), 915-928.

Hernández Galiot, A., \& Goñi Cambrodón, I. (2015). Calidad de la dieta de la población española mayor de 80 años no institucionalizada. Nutrición Hospitalaria, 31(6), 2571-2577. DOI:10.3305/nh.2015.31.6.8864

García-Esquinas, E., Graciani, A., Guallar-Castillón, P., López-García, E., Rodríguez-Mañas, L., \& Rodríguez-Artalejo, F. (2015). Diabetes and risk of frailty and its potential mechanisms: a prospective cohort study of older adults. Journal of the American Medical Directors Association, 16(9), 748754. DOI: https://doi.org/10.1016/i.jamda.2015.04.008 
Hardy, S.E., Perera, S., Roumani, Y.F., Chandler, J.M., \& Studenski, S.A. (2007). Improvement in usual gait speed predicts better survival in older adults. $J$ Am Geriatr Soc, 55(11), 1727-1734.

Hergenroeder, A.L., Brach, J.S., Otto, A.D., Sparto, P.J., \& Jakicic, J.M. (2011). The Influence of Body Mass Index on Self-report and Performance-based Measures of Physical Function in Adult Women. Cardpulm phy therapy J, 22(3), 11-20.

Higuita Gutiérrez, L. F., Arias, C., \& Antonio, J. (2015). Concept of quality of life in adolescence: a critical review of literature. CES Psicología, 8(1), 155158.

Hinton, P.S., Nigh, P., \& Thyfault, J. (2015). Effectiveness of resistance training or jumping-exercise to increase bone mineral density in men with low bone mass: A 12-month randomized, clinical trial. Bone, 79, 203-212. DOI: https://doi.org/10.1016/j.bone.2015.06.008

Lagiou, P., Trichopoulos, D., Sandin, S., Lagiou, A., Mucci, L., Wolk, A., \& Adami, H. O. (2006). Mediterranean dietary pattern and mortality among young women: a cohort study in Sweden. British Journal of Nutrition, 96(2), 384392. DOI: https://doi.org/10.1079/BJN20061824

Lera-López, F., Garrues Irisarri, M.A., Ollo-López, A.; Sánchez Iriso, E., Cabasés Hita, J.M., \& Sánchez Santos, J.M. (2017). Actividad física y salud autopercibida en personas mayores de 50 años / Physical Activity and Self-Perceived Health among People Aged 50 and Over. Revista Internacional de Medicina y Ciencias de la Actividad Física y el Deporte, 17 (67), 559-571. DOI: https://doi.org/10.15366/rimcafd2017.67.011

Mäkinen, T. E., Sippola, R., Borodulin, K., Rahkonen, O., Kunst, A., Klumbiene, J., \& Prättälä, R. (2012). Explaining educational differences in leisure-time physical activity in Europe: the contribution of work-related factors. Scandinavian journal of medicine \& science in sports, 22(3), 439447. DOI: 10.1111/j.1600-0838.2010.01234.x

Martí, A. Z., Cascales, R. F., Martínez, M. J. C., Sánchez, J. A. H., \& Pérez, A. L. (2015). Adherencia a la dieta mediterránea y su relación con el estado nutricional en personas mayores. Nutrición Hospitalaria, 31(4), 16671674. DOI:10.3305/nh.2015.31.4.8553

Martinez, A. (2016). Benefits of the Mediterranean diet beyond the Mediterranean Sea and beyond food patterns. BMC med, 14(1), 157. DOI: https://doi.org/10.1186/s12916-016-0714-3

Martínez, C., Veiga, P., Cobo, J., \& Carbajal, A. (2011). Evaluación del estado nutricional de un grupo de adultos mayores de 50 años mediante parámetros dietéticos y de composición corporal. Nutrición Hospitalaria, 26(5), 1081-1090. DOl: 10.3305/nh/2011.26.5.5.189

Matos-Duarte, M.; Martínez-de-Haro, V.; Sanz-Arribas, I.; Andrade, A.G.P., \& Chagas, M.H. (2017). Estudio longitudinal de la flexibilidad funcional en mayores físicamente activos / Longitudinal Study of Functional Flexibility in Older Physically Active. Revista Internacional de Medicina y Ciencias de la Actividad Física y el Deporte, 17 (65), 121-137. DOl: http://dx.doi.org/10.15366/rimcafd2017.65.008 
Nicholson, V. P., McKean, M. R., \& Burkett, B. J. (2015). Low-load high-repetition resistance training improves strength and gait speed in middle-aged and older adults. Journal of science and medicine in sport, 18(5), 596-600.

Norte, A.I., \& Ortiz, R. (2011). Calidad de la dieta española según el índice de alimentación saludable. Nutrición Hospitalaria, 26(2), 330-336.

Organización Mundial de la Salud (2010). Recomendaciones mundiales sobre actividad física para la salud. Catalogación por la biblioteca de OMS. Ginebra: Suiza.

Ortiz, R., Norte, A.I., Zaragoza, A., Fernández, J., \& Davó, M. (2012). ¿Siguen patrones de dieta mediterránea los universitarios españoles? Nutrición Hospitalaria, 27(6), 1952-1959. DOl: http://dx.doi.org/10.3305/nh.2012.27.6.6091

Papadaki, A., \& Scott, J. A. (2002). The impact on eating habits of temporary translocation from a Mediterranean to a Northern European environment. European Journal of Clinical Nutrition, 56(5), 455.

Possamai, L.T., Zogo, A., Boni, J., Jacques, M., Dorst, L.M., \& Dorst, D.B. (2015). Fitness for elders: A comparison between practioners and non-practioners of exercise. Age, 37(3), 1-8. DOI 10.1007/s11357-015-9772-2

Sallinen, J., Stenholm, S., Rantanen, T., Heliövaara, M., Sainio, P., \& Koskinen, S. (2010). Hand-grip strength cut points to screen older persons at risk for mobility limitation. Journal of the American Geriatrics Society, 58(9), 17211726. DOI: 10.1111/j.1532-5415.2010.03035.x

Scarmeas, N., Gu, Y., Schupf, N., Lee, J. H., Luchsinger, J. A., Stern, Y., \& Honig, L. (2014). Mediterranean diet and leukocyte telomere length in a multi-ethnic elderly population. Alzheimer's \& Dementia: The Journal of the Alzheimer's Association, 10(4), 755.

Schwingshackl, L., Hoffmann, G., Missbach, B., Stelmach-Mardas, M., \& Boeing, $\mathrm{H}$. (2017). An umbrella review of nuts intake and risk of cardiovascular disease. Current pharmaceutical design, 23(7), 1016-1027.

Sherrington, C., Fairhall, N., Kirkham, C., Clemson, L., Howard, K., Vogler, C., \& Sonnabend, D. (2016). Exercise and fall prevention self-management to reduce mobility-related disability and falls after fall-related lower limb fracture in older people: protocol for the RESTORE (Recovery Exercises and Stepping On after fracture) randomised controlled trial. BMC geriatrics, 16(34), 1-10

Talegawkar, S. A., Bandinelli, S., Bandeen-Roche, K., Chen, P., Milaneschi, Y., Tanaka, T., \& Ferrucci, L. (2012). A Higher Adherence to a MediterraneanStyle Diet Is Inversely Associated with the Development of Frailty in Community-Dwelling Elderly Men and Women, 2. The Journal of nutrition, 142(12), 2161-2166. DOI: https://doi.org/10.3945/jn.112.165498

Trichopoulou, A., Costacou, T., Bamia, C., \& Trichopoulos, D. (2003). Adherence to a Mediterranean diet and survival in a Greek population. New Eng $J$ Med, 348(26), 2599-2608.

Viñuales, I., Viñuales, M., Puzo, J., \& Sanclemente, T. (2016). Factores sociodemográficos asociados con el grado de adherencia al patrón de dieta mediterránea en personas mayores. Revista Española de Geriatría 
y Gerontología, 51(6), 338-341. DOl: https://doi.org/10.1016/j.regg.2015.09.017

Weening-Dijksterhuis, E., de Greef, M.H., Scherder, E. J., Slaets, J. P., \& Van der Schans, C.P. (2011). Frail institutionalized older persons: A comprehensive review on physical exercise, physical fitness, activities of daily living, and quality-of-life. American journal of physical medicine \& rehabilitation, 90(2), 156-168. DOI: 10.1097/PHM.0b013e3181f703ef

Zaragoza, A.M., Ferrer, R.C., Cabañero, M.M., Hurtado, J.S., \& Laguna, A.P. (2015). Adherence to the Mediterranean diet and its relation to nutritional status in older people. Nutrición hospitalaria, 31(4), 1667-1674. DOI: $\underline{10.3305 / \mathrm{nh} .2015 .31 .4 .8553}$

Número de citas totales / Total referentes: $39(100 \%)$

Número de citas propias de la revista / Journal's own referentes: $2(7,8 \%)$

Rev.int.med.cienc.act.fís.deporte - vol. 19 - número 76 - ISSN: 1577-0354 